\title{
The balance equation: Part 2. Derivation of the balance equation for response-specific inhibition
}

\author{
DOUGLAS ANGER \\ University of Missouri-Columbia, Columbia, Missouri
}

\begin{abstract}
This article and the preceding Part 1 (Anger, 1987) present a new molecular analysis of operant reinforcement and extinction. It is a multifactor analysis of the basic extinction processes that can be studied with procedures that minimize competing behavior. Part 1 showed that response-specific inhibition (RSI; Anger, 1983) can account for animal sensitivity to complex response contingencies. RSI depends on the same events that determine whether a reinforcer is contingent on a response. RSI can average those event frequencies, combine them into a single variable that varies with the contingency, and adjust responding appropriately. In Part 2 , the findings of Part 1 were used to derive a rational equation that describes operant responding. That linear additive equation specifies a balance between RSI-augmenting events (e.g., unreinforced responses) and RSI-decrementing events (e.g., reinforced responses). The equation predicts a different relation between responding and reinforcer frequency than do other theories, because it predicts that responding is augmented by an unrecognized variable, unreinforced nonresponse-time during controlling stimuli.
\end{abstract}

This article continues the analysis of Part 1 (Anger, 1987), whose prior reading will probably be needed, although major abbreviations are restated here. The term $P$ represents a response, and $P^{\prime}$ represents the absence of that response during the stimulus in whose presence $P$ is sometimes reinforced, hereafter called $\mathrm{S}+$. A reinforcer is represented by $r$, and reinforcer absence by $r^{\prime}$. Then $P, r$ refers to a $P$ closely followed by an $r ; P, r^{\prime}$ to a $P$ not closely followed by an $r$; $P^{\prime}, r$ to an $r$ that occurs after nonoccurrence of $P$ during an $\mathrm{S}+$ (reinforcement of RSI); and $P^{\prime}, r^{\prime}$ to the occurrence of $\mathrm{S}+$ without either $P$ or $r$. Sequence is used as the group name for $P, r ; P, r^{\prime} ; P^{\prime} r$ and $P^{\prime}, r^{\prime}$. Expressions without brackets refer to individual cases of the sequences; when bracketed (e.g., $\left.\left[P, r^{\prime}\right]\right)$, they refer to the rate of occurrence of the sequence per hour (or other time unit of the period studied).

In Part 1 it was proposed that response-specific inhibition (RSI) is increased or decreased by each of the four sequences; consequently RSI both averages their frequencies and combines them into a single variable, which assesses the contingency and adjusts the response frequency appropriately. Let us now restate that proposition more precisely as an equation. For contingency detection, such an equation should have the following properties: (1) Each instance of a sequence should have a certain effect on RSI independent of the frequency of the other sequences. Hence, an additive relation between the effects of the different sequences is implied, rather than a multiplicative one. (With a multiplicative rela-

This research was funded in part by a grant from the Research Council of the Graduate School, University of Missouri-Columbia. This analysis was presented at the Association for Behavior Analysis meeting in Columbus, $\mathrm{OH}, 1985$. The author wishes to thank Kathleen Anger, Lynn Hammond, Eliot Hearst, Richard Herrnstein, Andrew Homer, Duncan Luce, Marjorie Marlin, William Timberlake, Trish Vandiver, Edward Wasserman, and Raymond Wolf for valuable comments. Reprints may be obtained from the author, Department of Psychology, McAlester Hall, University of Missouri, Columbia, MO 65211. tion, the effect of one instance of a sequence depends greatly on the frequency of the other sequences.) (2) To average the frequencies of each type of sequence, individual instances of a sequence should have the same effect independent of the frequency of that sequence. To obtain that independence, a linear term is needed for each sequence. Consequently, the earlier proposition implies the following additive linear equation in which $\left[P^{\prime}, r\right]$ and $\left[P, r^{\prime}\right]$ have effects opposite those of $[P, r]$ and $\left[P^{\prime}, r^{\prime}\right]$ :

$$
\mathrm{D}[P, r]+\mathrm{E}\left[P^{\prime}, r^{\prime}\right]-\mathrm{F}\left[P, r^{\prime}\right]-\mathrm{G}\left[P^{\prime}, r\right]=0 .
$$

The four constants, D, E, F, and G, depend on the relative effects of the four sequences, which in turn depend on the procedure. Thus, $\mathrm{D}$ and $\mathrm{G}$ for $[P, r]$ and $\left[P^{\prime}, r\right]$ depend on $r$ variables, such as the size and nature of $r$, and $\mathrm{F}$ for $\left[P, r^{\prime}\right]$ depends on $\boldsymbol{P}$ variables. These constants will initially have to be determined empirically, but the same constants should be found across changes in procedure when the $P$, the $r$, and other relevant variables are held constant.

This equation seems to incorporate the earlier principles quite directly, and seems to be the simplest way to implement them. It will be called the balance equation, because it specifies only the situation when the RSI-augmenting effects of $\left[P, r^{\prime}\right]$ and $\left[P^{\prime}, r\right]$ balance the RSI-decrementing effects of $[P, r]$ and $\left[P^{\prime}, r^{\prime}\right]$, so that the behavior is stable. When the equation does not balance, then RSI either increases or decreases, hence $[P]$ either decreases or increases, until the $[P]$ change restores balance or until further $[P]$ change is limited by other factors, such as zero or maximum $[P]$. When $[P, r]$ increases to the point at which $[P]$ reaches $100 \%$ or its maximum level and all latencies are short, then further increases in $[P, r]$ can no longer be balanced by increases in $\left[P, r^{\prime}\right]$ or by decreases in $\left[P^{\prime}, r^{\prime}\right]$. Then the equation cannot be balanced, and the RSI is zero. Such imbalance occurs with a variety of $[P, r]$ values, and for them the only prediction is maximal $[P]$. Similarly, when increase in $\left[P^{\prime}, r\right]$ reduces 
$[P]$ to zero, then further increases in $\left[P^{\prime}, r\right]$ can decrease $[P]$ no further, and the equation can no longer be balanced. Then the only prediction is zero $[P]$.

The balance equation also does not describe transitions between two stable states. Additional assumptions (e.g., that the rate of approach is proportional to the deviation from the stable state) may provide a basis for describing transitions, but that possibility will not be pursued in this report. The balance equation only describes stable behavior when $[P]$ is neither $100 \%$ nor $0 \%$.

The Probability Difference measure was discussed in Part 1 and is repeated below:

$$
\frac{[P, r]}{[P, r]+\left[P, r^{\prime}\right]}-\frac{\left[P^{\prime}, r\right]}{\left[P^{\prime}, r\right]+\left[P^{\prime}, r^{\prime}\right]} \text {. }
$$

The discussion of this measure and the balance equation was in terms of a trial procedure, because a trial procedure eliminates the wide variety of temporal spacings between $P$ and $r$ that can occur with the free operant. Instead, a trial procedure allows only the two extreme spacings: a $P$ followed immediately by an $r$, and a $P$ essentially not followed by an $r$, because the temporal separation is sufficient to eliminate any appreciable effect of the $r$. The trial procedure will be continued in our development of the balance equation. However, the use of the Probability Difference also required measurement of $\left[P^{\prime}, r^{\prime}\right]$ as a count of trials with $P^{\prime}, r^{\prime}$. Time measurements could not be used for $\left[P^{\prime}, r^{\prime}\right]$, because if they had been used, then no matter what the value of the other three terms, the Probability Difference could have been made zero or large simply by changing the time units chosen for measurement of $\left[P^{\prime}, r^{\prime}\right]$. However, in the balance equation, time units for $\left[P^{\prime}, r^{\prime}\right]$ present no problem, because in the balance equation $\left[P^{\prime}, r^{\prime}\right]$ is multiplied by a constant ( $\mathrm{E}$ in Equation 1), which depends on procedural factors, and that constant can include a constant for the time units used in the measurement of $\left[P^{\prime}, r^{\prime}\right]$. (Measurement of $\left[P^{\prime}, r^{\prime}\right]$ in minutes simply results in an $E$ value 60 times larger than when measured in seconds.) When we apply this equation to actual experimental procedures, numerous problems arise that make necessary the measurement of $\left[P^{\prime}, r^{\prime}\right]$ in time units. For example, RSI and $\boldsymbol{P}^{\prime}, \boldsymbol{r}^{\prime}$ occur not only on trials with no $P$ and no $r$; they also occur on trials with a long latency $P$ (i.e., when the animal inhibits $\boldsymbol{P}$ for a while before responding). It will also be found that the $\mathrm{S}+$ time for the rat may not be the entire bar-available time, but a shorter interval that has to be measured. For these and other reasons, time units will now be used for the measurement of $\left[P^{\prime}, r^{\prime}\right]$ in the balance equation.

Due to its balancing nature, all effects are relative in this equation, so one constant can be eliminated by expressing the other three as multiples of it. Many schedule results show that one $P, r$ balances many $P, r^{\prime}$ s, so usually the effect of a $P, r^{\prime}$ will be weaker than a $P, r$ or a $P^{\prime}, r$. Hence, the constants for $[P, r]$ and $\left[P^{\prime}, r\right]$ will usually be greater than 1.0 if expressed in terms of $\left[P, r^{\prime}\right]$. Since the $\left[P^{\prime}, r^{\prime}\right]$ constant will probably be the least accurately measured one, it seems best not to express the others in terms of $\left[P^{\prime}, r^{\prime}\right]$. Thus, with transposition we have

$$
\mathrm{A}[P, r]+\mathrm{B}\left[P^{\prime}, r^{\prime}\right]=\left[P, r^{\prime}\right]+\mathrm{C}\left[P^{\prime}, r\right] .
$$

In this form the effect of one $P, r^{\prime}$ becomes the unit of measurement of the effect of the other three sequences, and the meaning of the constants becomes easy to grasp: the constant A specifies how many $P, r^{\prime}$ s are required to balance the effect of one $P, r$; the constant B specifies how many $P, r^{\prime}$ s are required to balance the effect of one second of $P^{\prime}, r^{\prime}$, and so forth.

Balance equation 3 is surprisingly simple and parsimonious. Three parametric constants are the minimum possible with four different behavioral effects, since at least three of the effects are known to depend on other variables. It is possible for the experimenter to minimize $\left[P^{\prime}, r\right]$, and in extinction $[P, r]$ is eliminated, so by such manipulations the constants can be determined. This equation is not an ad hoc equation fitted to data, but instead is rationally derived from basic findings and the nature of the response-reinforcer contingency.

The linear additive equation also seems the best equation for the initial exploration of the balance model, because it involves the fewest assumptions. It proposes that each instance of a sequence, say each $P, r^{\prime}$, has the same effect as every other $P, r^{\prime}$, independent of $\left[P, r^{\prime}\right]$ or the rates of the other sequences. Other equations would involve the assumption of additional effects and constants without adequate evidence for the presence or character of such effects. If nonlinear terms are used for the effect of sequences, then an instance of $P, r$ has a different effect at high $[P, r]$ than at low $[P, r]$, and we need to specify the nature and constants of that variation. If an equation is used that combines the sequences by multiplication and division, then the relation between the effects of two sequences, say $P, r$ and $P, r^{\prime}$, is highly dependent on the frequency of the other sequences, and we need to specify the nature and constants of that dependence. It seems best to start with the assumption of no such effects, and add effects only after we have evidence for their presence and character. Zero $\left[P^{\prime}, r\right]$ is common, and zero $[P, r]$ occurs in extinction. Such zeros generate problems for a multiplicative relation, since zeros in the numerator cause the whole expression to become zero, and zeros in the denominator are not permitted.

The term RSI is used for a process that is considered either active or inactive. When RSI is active during an $\mathrm{S}+$ for that $P$, then no $P$ occurs, and when inactive, $P \mathrm{~s}$ occur. That definition facilitates the measurement of RSI: the RSI time is $P^{\prime}, r^{\prime}$ during an $\mathrm{S}+$. However, when we speak of $P, r^{\prime}$ augmenting RSI or of $P, r$ decrementing RSI, we are referring to an increase or decrease in the frequency of RSI relative to $P$ during the $\mathrm{S}+$, a variable that will be called the RSI strength to distinguish it. At present, the most suitable measure of RSI strength seems to be the proportion of RSI time to non-RSI time, that is, the ratio of RSI time to $P$ time. However, until we know more about the duration of $P$, it seems better to use the more easily measured count of $P$, which should be sufficiently proportional to the $P$ time for our present purposes. Thus $\left[P^{\prime}, r^{\prime}\right] /[P]$ will be used to estimate RSI strength. It measures the effectiveness of RSI in eliminating $P$ s over a 
period of time, and is independent of sample size. If applied to a free operant procedure, it approximately equals the mean interresponse time, or IRT. (The equality is approximate, because $\left[P^{\prime}, r^{\prime}\right]$ excludes the time required for a $P$, and the IRT does not, a difference only important at high $[P]$.) Since the mean IRT is the reciprocal of the response rate, the basic behavior measure of this analysis approximates the reciprocal of the widely used response-rate measure, although it is viewed somewhat differently, as a measure of average RSI.

Although balance equation 3 specifies the factors that augment and decrement RSI strength and the conditions under which they balance, a term for RSI strength does not appear in that equation. However, the following expression for RSI strength is easily obtained from balance equation 3 by dividing it by $\mathrm{B}[P]$ and rearranging:

RSI strength $=\frac{\left[P^{\prime}, r^{\prime}\right]}{[P]}=\frac{\left[P, r^{\prime}\right]}{\mathrm{B}[P]}+\frac{\mathrm{C}\left[P^{\prime}, r\right]}{\mathrm{B}[P]}-\frac{\mathrm{A}[P, r]}{\mathrm{B}[P]}$

On the one hand, Equation 4 is just a more complex version of the balance equation describing the input conditions that balance and produce stability at intermediate levels of responding. On the other hand, Equation 4 simultaneously provides two expressions for the RSI strength, two different measurable quantities: (1) $\left[P^{\prime}, r^{\prime}\right] /[P]$, which we have already discussed, and $(2)\left[P, r^{\prime}\right] / \mathrm{B}[P]+\mathrm{C}\left[P^{\prime}, r\right] / \mathrm{B}[P]-$ $\mathrm{A}[P, r] / \mathrm{B}[P]$. These two expressions are equal when balance occurs. In Equation 3, $\left[P^{\prime}, r^{\prime}\right]$ was viewed as an input into RSI strength, but in the above discussion and in Equation 4, it is treated as an output of the animal. Actually this dual role as both input and output is true for all four sequences, because all four contain either a $P$ or a $P^{\prime}$, the two outputs of the animal, and all four contain an $r$ or an $r^{\prime}$, the two inputs from the experimenter. We ordinarily think of $[P, r]$ and $\left[P^{\prime}, r\right]$ as input variables under the experimenter's control, and that is the usual situation when we reinforce only a small fraction of the $P$ s that occur, so we can deliver $P, r$ and $P^{\prime}, r$ pretty much whenever we choose. However, when we try to reinforce an extremely low frequency response or a certain latency, then we sometimes are helpless when that response does not occur at the times when we wish to reinforce it. Then we realize that sometimes $[P, r]$ and $\left[P^{\prime}, r\right]$ can be very much under the animal's control.

On the other hand, for $P, r^{\prime}$ and $P^{\prime}, r^{\prime}$ the output aspects are conspicuous, and their input aspects (the experimenters doing nothing, providing no reinforcers) are easily overlooked. However, the experimenters doing nothing is a very important input to the animal. This RSI analysis emphasizes that both $P^{\prime}, r^{\prime}$ and $P, r^{\prime}$ are inputs to the animal, and that both, along with $P, r$ and $P^{\prime}, r$, change RSI strength too. All four output sequences have to be inputs if the animal is sensitive to complex contingencies, although under usual conditions, the experimenter has more control of $[P, r]$ and $\left[P^{\prime}, r\right]$ and the animal has more control of $\left[P, r^{\prime}\right]$ and $\left[P^{\prime}, r^{\prime}\right]$.

It may be helpful here to point out the difference between the balance equation analysis and a related group of important investigations that will be discussed more fully by Anger (1988). Those studies were concerned with demonstrating and estimating the effect of contingency on behavior. That effort encounters the problem that with most operant proce- dures and contingency measures, changes in behavior affect the measure of contingency (Gibbon, Berryman, \& Thompson, 1974). However, two studies have developed procedures that stabilize a measure of the contingency applied to the animal independent of the animal's behavior. Hammond (1980) used a free operant procedure that divided the session into 1-sec time intervals and programmed a constant conditional probability of $r$ given that a $P$ occurred in an interval, and independently a constant conditional probability of $r$ given that $P^{\prime}$ occurred in an interval. Those applied conditional probabilities and their difference did not change with changes in the responding, and the rats showed good sensitivity to changes in the Probability Difference. Scott and Platt (1985) developed a different procedure, which stabilized a different contingency measure, phi, in the presence of behavior changes. They, too, showed major effects on rat behavior of changes in their applied contingency measure.

Those experiments succeeded in their important objective of demonstrating the sensitivity of animals to environmental contingencies, but they did not address the question of what processes were responsible for the sensitivity, and did not propose that animals were reacting to the measures they used. Scott and Platt said, "It is implausible that rats are doing anything like computing phi values over sessions" (1985, p. 169). In contrast, the present analysis used complex measures that are influenced by both the environment and behavior, because those appear to be the inputs available to the animal, and the objective is to determine how the animal's inputs and conditioning processes can adjust the animal's behavior to the applied contingency. In brief, the above investigators found evidence that animals do it, whereas the balance equation is a proposal about how they do it. It seems well within the capacity of the vertebrate nervous system to carry out the operations implied by the balance equation, that is, to generate a variable that controls response frequency, with that variable augmented by certain inputs, decremented by other inputs, and hence stabilized when those inputs balance.

Although the role of contingency in behavior is a major source of the balance equation, its balancing of augmenting and decrementing factors also fits the often conspicuous stability of behavior. Behavior seems to be stabilized by negative feedback when perturbed by fluctuations or when variables are deliberately changed.

For example, on a variable-interval schedule (VI), if $[P]$ fluctuates upward due to some uncontrolled event, then $\left[P, r^{\prime}\right]$ increases, which has effects that decrease $[P]$ and counteract the initial $[P]$ increase. This negative feedback also suggests a balancing of augmenting and decrementing factors, and the balance equation incorporates those feedback features. For the just mentioned upward fluctuation of $[P]$, the increase in $[P]$ increases $\left[P, r^{\prime}\right]$ on the right side of Equation 3, which increases the RSI strength, which causes a decrease in $[P]$, thereby counteracting the initial increase in $[P]$, and rebalancing the equation at the same level as before. (In the usual VI schedule, $[P]$ and $\left[P, r^{\prime}\right]$ are approximately equal.)

Or suppose that with the VI schedule, the experimenter increases $[P, r]$, which decreases RSI strength. Then $[P]$ increases until the resulting higher $\left[P, r^{\prime}\right]$ rebalances the equation. (A small decrease in $\left[P^{\prime}, r^{\prime}\right]$ also occurs, but its effect 
would be small for VI.) At the new balance point, $[P]$ is higher and $\left[P^{\prime}, r^{\prime}\right]$ is lower; hence $\left[P^{\prime}, r^{\prime}\right] /[P]$, the RSI strength, is lower. If the experimenter increases $\left[P^{\prime}, r\right]$, then the RSI strength increases, which decreases $\left[P, r^{\prime}\right]$, and increases $\left[P^{\prime}, r^{\prime}\right]$, both of which decrease RSI strength, and rebalance the equation again at a lower $[P]$, hence at a higher $\left[P^{\prime}, r^{\prime}\right] /[P]$, a higher RSI strength.

If we increase the $\left[t_{\mathrm{s}+}\right]$ without changing the $[P, r]$ in the sample, a more complex change results. The increased $\left[t_{s+}\right]$ increases $\left[P^{\prime}, r^{\prime}\right]$, which will decrease the RSI strength, which will produce a compensating increase in $\left[P, r^{\prime}\right]$, which rebalances the equation. However, the RSI strength, $\left[P^{\prime}, r^{\prime}\right] /[P]$, at the new balance will not necessarily be the same as before. For example, let us simplify by making $\left[P^{\prime}, r\right]=0$, so Equation 3 becomes

$$
\mathrm{A}[P, r]+\mathrm{B}\left[P^{\prime}, r^{\prime}\right]=\left[P, r^{\prime}\right] .
$$

To simplify further, consider the case where the $\mathrm{A}[P, r]$ and $\mathrm{B}\left[P^{\prime}, r^{\prime}\right]$ terms happen to be initially equal. Then doubling $\left[P^{\prime}, r^{\prime}\right]$ with $[P, r]$ constant will only increase $\left[P, r^{\prime}\right]$ by $50 \%$, because we have doubled only one of the two terms, $\mathrm{A}[P, r]$ and $\mathrm{B}\left[P^{\prime}, r^{\prime}\right]$, in Equation 5 that contribute equally to decreasing RSI strength. Hence $\left[P, r^{\prime}\right]$, the only term in Equation 5 that increases RSI strength, is not doubled, but only increases $50 \%$. When $\left[P^{\prime}, r^{\prime}\right]$ is doubled and $\left[P, r^{\prime}\right]$ (which usually approximates $[P])$ is only increased $50 \%$, then $\left[P^{\prime}, r^{\prime}\right] /[P]$, our measure of RSI strength, would increase! At first it seems surprising that increasing a sequence, which by itself decreases RSI strength, has a final effect of increasing RSI strength. It does so because the equation describes a complex feedback system, and we have increased $\left[P^{\prime}, r^{\prime}\right]$ but not $[P, r]$. Since $\left[P, r^{\prime}\right]$ equals the sum of $[P, r]$ and $\left[P^{\prime}, r^{\prime}\right]$ effects, the percentage increase in $\left[P, r^{\prime}\right]$ is less than the percentage increase of $\left[P^{\prime}, r^{\prime}\right]$. If we had doubled $[P, r]$ along with doubling $\left[P^{\prime}, r^{\prime}\right]$, then $\left[P, r^{\prime}\right]$ would have doubled too, and RSI strength would have stayed constant, since in that case both numerator and denominator of $\left[P^{\prime}, r^{\prime}\right] /[P]$ would have been doubled. In summary, the balance equation specifies several different negative feedback effects that seem to fit the complex stability and adjustment to change that we find in operant behavior.

The attractive features of the balance equation are that it is simple and rational, yet it has subtle features that enable it to fit a variety of complex situations. Although the concept of RSI has played a major role here in the development of the equation, a rational case for the balance equation can be made without the RSI concept, and with few assumptions. One starts from the empirical effects of $P, r ; P, r^{\prime}$; and $P^{\prime}, r$ on $[P]$, with no assumption about how they act, whether by RSI, competing responses, or whatever. Somehow these effects must combine to control $[P]$, since all three affect $[P]$. The balance equation has the fewest constants and makes the fewest assumptions about how that combination occurs; it assumes uniform effect without interactions. Possibly the effect of one sequence may change with changes in its own frequency or the frequency of other sequences, but until such complicating effects are demonstrated, Occam's razor suggests that first we try the linear additive equation and not increase the number of effects unnecessarily. However, just from an empirical standpoint, it seems wise to determine whether $P^{\prime}, r^{\prime}$ might also have an effect on $[P]$, and, if it does, then it should also be included in the balance equation. This argument for investigating the balance equation seems only dependent on the secure empirical evidence that $P, r ; P, r^{\prime}$; and $P^{\prime}, r$ all affect $[P]$, and is independent of the proposal of RSI and contingency detection by animals. However, if one grants that animals can adjust their responding appropriately to complex contingencies, then it follows that animals must be influenced by $P^{\prime}, r^{\prime}$ also, and the balance equation provides an explanation for how the adjustment to contingency is produced by a single variable averaging and combining the effects of the four sequences. Then the concept of RSI, for which there is independent evidence (Anger, 1983), fits nicely as the variable that does the averaging and combining.

The above analysis demonstrates the importance of investigating the balance equation, but its real test will be how well experiments confirm its quantitative predictions about behavior. In that respect, the balance equation makes many specific and quantitative predictions, some of them quite surprising and different from other theories. Such critical tests of the equation will be discussed in a later article (Anger, 1988). If such tests support the equation, they will generate additional information about RSI, which will make possible the application of the equation to more complex procedures.

The problem with the balance equation is that other factors besides RSI influence responding and obscure the RSI effects. Were it otherwise, no doubt the balance equation would have been recognized long ago. These complicating factors, special procedures that avoid these factors, and preliminary results will be described in another article (Anger, 1988). That article will also examine the relation of the balance equation and the multifactor theory to other theories and research.

\section{REFERENCES}

ANGER, D. (1983). Reinforcement of inhibition. Journal of the Experimental Analysis of Behavior, 39, 213-226.

ANGER, D. (1987). The balance equation: Part 1. Response-specific inhibition and the operant-contingency puzzles. Bulletin of the Psychonomic Society, 25, 468-471.

ANGER, D. (1988). The balance equation: Its testing and relations to other research. Manuscript in preparation.

Gibbon, J., Berryman, R., \& ThOmpson, R. L. (1974). Contingency spaces and measures in classical and instrumental conditioning. Journal of the Experimental Analysis of Behavior, 21, 585-605.

HAMmoND, L. J. (1980). The effect of contingency upon the appetitive conditioning of free operant behavior. Journal of the Experimental Analysis of Behavior, 34, 297-304.

ScotT, G. K., \& PlatT, J. R. (1985). Model of response-reinforcer contingency. Journal of Experimental Psychology: Animal Behavior Processes, 11, 152-171.

(Manuscript received for publication April 23, 1987.) 